\title{
Muscle Electromyography Analysis of Upper Arm during Dragon Boat Paddling
}

\author{
Yabin $\mathrm{He}^{1}$, Guoqin Jiang ${ }^{1}$, Yuxiang $\mathrm{Wu}^{1, \text { * }}$ \\ ${ }^{1}$ School of Physical Education, Jianghan University, Wuhan 430056, China; \\ *Author for correspondence: 408328331@qq.com
}

Keywords: dragon boat, paddle, EMG

\begin{abstract}
Dragon boat sport are the traditional Chinese cultural and sports projects, the integration of national cultural connotation, mass fitness and modern athletic characteristics, is rapidly moving towards international and modern road. In order to further understand and improve the technical characteristics and laws of dragon boat paddle, this paper uses the experimental research method to carry on the wireless electromyography telemetry to the university student outstanding dragon boat athlete, through the entire paddle cycle technical movement research, and analyzes its related muscle surface muscle Electric characteristics of the dragon boat to improve the teaching and special training to provide theoretical basis and reference. The results showed that there was a significant difference in the degree of muscular exertion and the relative muscle position in the same and opposite sides of the paddle during the paddling cycle, and the strength of the upper limb muscle was higher. In addition, the core part of the muscle group is also the completion of the entire paddle action has an important role cannot be ignored.
\end{abstract}

\section{Introduction}

In the historical development of China, the Chinese nation regards the dragon as a totem in the hearts of every person. It is a spiritual sustenance, a heartfelt statement of blessing and prayer, and a symbol of the Chinese people's diligence, courage, initiative, perseverance and self-reliance. Dragon Boat races for thousands of years as an important custom in the land of China heritage, has been deeply loved by people of all nationalities.

As an important folk traditional Chinese sports, dragon boat racing has gone beyond the scope of sports, has become the Chinese nation's inner cultural spirit of the performance. It is a manifestation of the dragon culture is the carrier of the spirit of the Chinese nation is one of the characteristics of the direct expression of national characteristics, is an important national traditional sports culture. Chinese dragon culture elements of the classic performance of the world culture have had an important impact.

Modern dragon boat race has changed from the past folk performances into a sports competition. In the past ten years, with the development of the sport's growth, game time is no longer confined to the day of the Dragon Boat Festival, the prize is more and more high, the level of organization is more and more high, which makes the strength of the team have more chances. Plus all kinds of media are also actively involved in the game, for the game, so that more and more sponsors to see the business opportunities contained in them, so that the professionalization of the dragon boat sports have become possible.

Sponsored by the national sports administrative guidance center, Chinese Dragon Boat Association and CCTV sports channel cooperation and the Chinese Dragon Boat Race Dragon boat tournament since 2011. After the promotion and development of 4 years, the dragon boat Sport watch and competition get a full range of ascension and the dragon boat sport and dragon boat club is booming. The inheritance and development of the dragon boat movement will play an important role in the transmission of the traditional culture of the Chinese nation and the promotion of the national fitness program. 


\section{Method}

In this study, Wave Plus 16 channel EMG measurement system was used for wireless telemetry of athletes in outdoor water environment. The sampling frequency was $1000 \mathrm{~Hz}$.The research object is the Jianghan University dragon boat team 10 right paddle a dragon boat team. The sEMG signal of the musculus biceps brachii and musculus triceps brachii during the rowing process was recorded, and the sEMG signal was recorded in the electromyography time-domain index Integral electromyography (IEMG) for statistical analysis. EMG data analysis was performed by EMG Easy Report analysis software.

In the dragon boat race, paddle belonging to cyclical movements, economy and effectiveness of its action has a very important effect on the performance and motivation are based on dragon boat rowing water on the blade's reaction, improve the stability and effectiveness of oarsmanship chain, can effectively improve the athletes of every paddle. Effect. Through the study of the characteristics of the electrical activity of the muscles of the paddle can better determine the features of its technical characteristics is scientific and is in accordance with the biomechanical kinematic chain. Surface electromyography technology can be very good to the biological changes of neuromuscular activity process guidance, amplified and recorded, on muscle, coordination degree, contraction strength and fatigue degree evaluation results, have become indispensable in the field of sports medicine research means. The college student dragon boat athletes as the research object, through the real water environment on the paddle muscles for wireless telemetry, EMG characteristics of muscles were recorded, in order to provide theoretical basis and reference for teaching and training in the future of dragon boat sports.

Table 1 . Basic physical quality characteristics of dragon boat athletes

\begin{tabular}{cccc}
\hline Age(year) & Height $(\mathrm{cm})$ & Weight(kg) & $\begin{array}{c}\text { Maximum } \\
\text { bench press(kg) }\end{array}$ \\
\hline $21.4 \pm 1.1$ & $182.4 \pm 7.0$ & $76.6 \pm 5.6$ & $102.5 \pm 10.8$ \\
\hline
\end{tabular}

\section{Results}

Integrated Electromyogram (Integrated Electromyogram, IEMG) refers to a certain period of time, all involved in activities, the size of the total muscle discharge. It reflects to a certain extent the number of units involved in the activities of the number of units and the discharge of each unit of force, thus representing the paddle muscle activation of the degree of force. When the absolute value of the signal is taken, noise will make the mathematical integral have a constant increase. Integrated EMG splits up the signal into fixed-width time slices and resets the integral at the start of each time slice. 


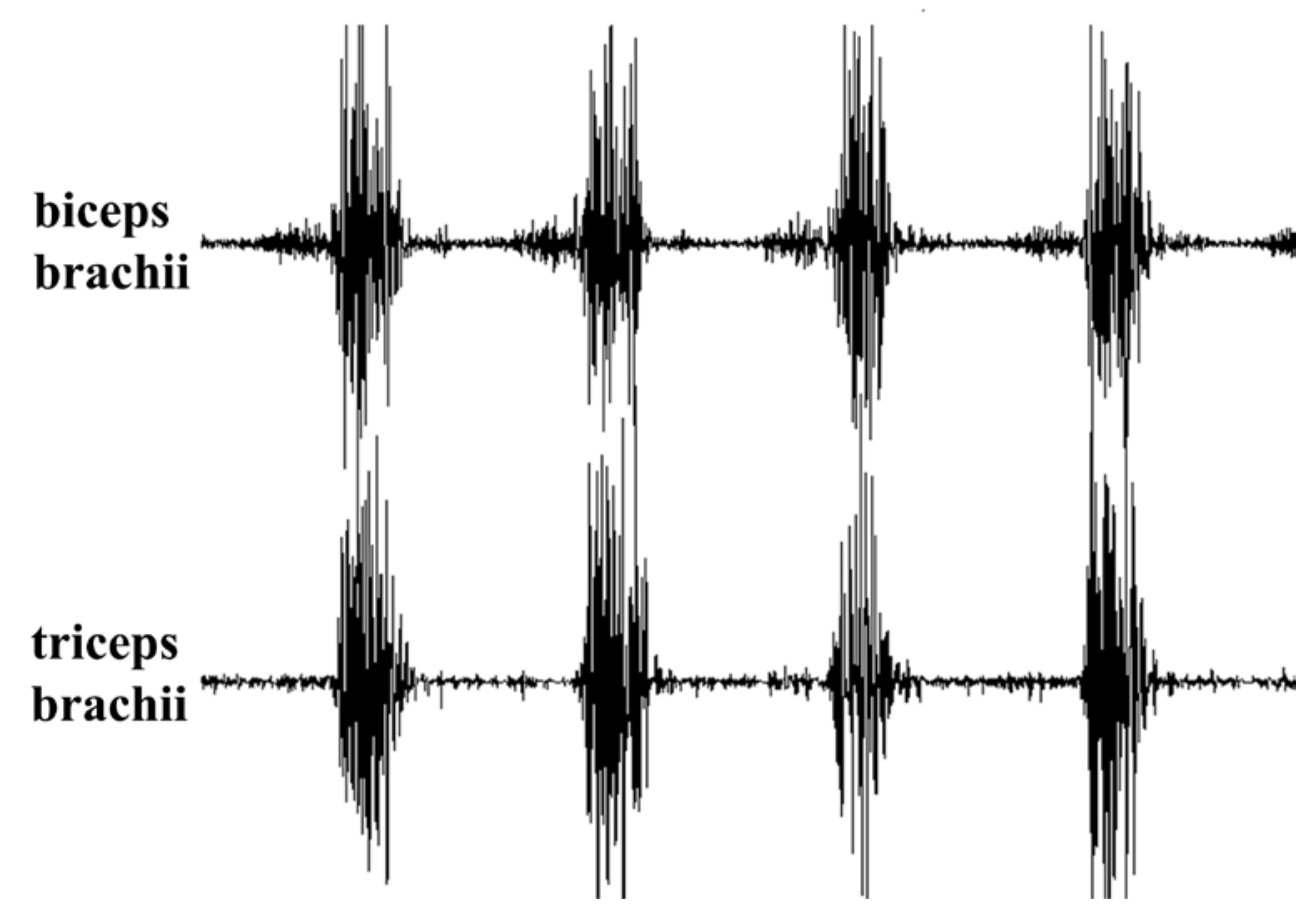

Fig. 1 Real-time recording of EMG signals during the paddling of dragon boat.

The results of figure 1 showed the speed of the action is highlighted on the basis of the athlete's technical training, and the special technical force of the continuous work is completed by overload, while maintaining the integrity of the technology. Along with raising the level of athletic, the athlete's strength level requirements are increasingly high, the past through the development of muscle strength is difficult to improve the athlete's special forces required, according to the structural characteristics of dragon boat movement technology force, the development of core strength athletes, to improve the athletes' physical ability level is essential.

Table 2. IEMG of different parts of muscles

\begin{tabular}{cc}
\hline Muscle & IEMG \\
\hline biceps brachii & $214.2 \pm 15.3$ \\
triceps brachii & $209.6 \pm 13.9$ \\
\hline
\end{tabular}

The dragon boat sport is a high load and high strength of the national traditional sports project, the project characteristics of dragon boat, dragon boat on the physical fitness of athletes have higher requirements, the physical training of the athletes must reflect the characteristics of special physical training, according to the special action of technical training, each item of the athletes' physical fitness requirements are not the same, the use of the muscles the action in the process, the body is not the same "system to develop different joint angles under the action force, movement speed, muscle contraction speed should be consistent with the special action at the same time, pay attention to the standardization of technical movements. Physical training load should meet the requirements of the load conditions of competition, make the body energy metabolism and competition, higher than the competition strength training means, can effectively stimulate the body to produce appropriate physiological adaptation, in order to maximize the special athletic ability. According to the characteristics of the dragon boat sport, physical training should be training on the water and land training combination, can play a better role in development so that to improve the special level of dragon boat athletes and the overall quality of the land, only for water use, with land and water, in order to achieve better training effect. 


\section{Discussion and Conclusion}

Dragon boat race entered the Asian Games in 2010, marking the dragon boat movement into the international comprehensive games. This series of international competitions marked the dragon boat movement into the ranks of international sports. In recent years, the dragon boat sport has been widely carried out in the world, the world of dragon boat has improved quickly, Europe and some Southeast Asian countries, the dragon boat competition level has reached the world advanced level, whereas in the international competition gold medal, the competition is fierce, in order to improve the match results, gradually increase in the project of scientific research the technical level of investment, the project is also rising. Dragon boat, a traditional Chinese national sport, is widely spread and developed all over the world.

Dragon boat race is a traditional sports with a long history of thousands of years, its deep cultural heritage and unique charm by the people of the world's identity and love. The development of modern dragon boat racing is both entertaining and competitive, so that it can spread to all parts of the world at a faster speed. Now it has become an international sport. In this study, Jiangnan University excellent dragon boat athletes throughout the paddling cycle of technical action research, analysis of the relevant muscle surface EMG characteristics parameters.

Dragon boat race is a high intensity and high load sports. It is not only a traditional Chinese folk sports, but also the integration of modern sports elements. Only when we correctly grasp the characteristics of the dragon boat race, learn from the scientific training theory, and improve the scientific training level, we could make the dragon boat movement towards the direction of internationalization and modernization.

\section{Acknowledgments}

The work was supported by Natural Science Foundation of Hubei Province (Grant No. 2016CFB327) and Foundation of Wuhan Sports Bureau (Grant No. 2016Z018). Author for correspondence: 408328331@qq.com.

\section{References}

[1] R. Sarah, M. Richard ,G. Philip ,J. Peter, Physiological and Physical Characteristics of Elite Dragon Boat Paddlers, Journal of Strength \& Conditioning Research.27(2013)137-145.

[2] M. Brooke, Fongzi, dragons and corporate culture: An analysis of corporate dragon-boat paddlers, Journal of Sport and Social Science. 4(2015)99-112.

[3] A. Chittick, Competitive Spectacle during China's Northern and Southern Dynasties: With Particular Emphasis on Dragon Boat Racing, Asia Major. 23(2010)65-85.

[4] Z. Liang, L. Wei, L. Daoming, Interactional Research on Fujian Dragon-boat Culture and the Construction of West-strait Economic Zone, Journal of Hebei Institute of Physical Education. 24(2010)84-86.

[5] Z. Yaoyao, Practical Analysis and Discussion on the Tactical of Competitive Dragon Boat Race of 16th Asian Games, A Thesis Submitted for the Degree of Master. Wuhan Institute of Physical Education, 2012.

[6] T. Licheng, The Development Status of Dragon Boat Racing and Countermeasures Analysis, Friend of Science Amateurs. 4(2010)140-142.

[7] L. Bing, Dragon training in China Construction and development of scientific research base, Journal of Northeast Dianli University. 30(2010)89-92.

[8] J. Yuliang, Research on Dragon Boat Races in Xiangyang, Journal of Hubei University of Arts and Science. 33(2013)41-45. 